\title{
5 Nicht-menschliche Kommunikation und die Komplexität natürlicher Sprachen
}

[L]anguage doesn't look as if it should be all that complex, not like genetics or quantum mechanics. We all speak at least one, that one we acquired without a lick of conscious effort, and most non-linguists, in the unlikely event that they opened a copy of Linguistic Inquiry or Natural Language and Linguistic Theory only to find stuff every bit as hard going as genetics or quantum mechanics, would in many cases react by saying „What's all this nonsense about? Why are they making such a fuss about something that's perfectly simple and straightforward?“ And they would probably go on to say, „What do I need this stuff for? I'm a systematic biologist/paleoanthropologist/evolutionary psychologist/computa-tional mathematician [strike out whichever don't apply]--I don't need this.“

Bickerton (2003: 77)

\subsection{Die Rolle der Sprachwissenschaften in der einschlägigen Forschung}

Bildet erstens die moderne Evolutionstheorie den Rahmen und Hintergrund der Evolution menschlicher Sprachfähigkeit und strukturieren zweitens die Erkenntnisse der Paläoanthropologie mitsamt der Archäologie den Verlauf dieser Entwicklung anhand von Physiologie, Technologie und Verhalten, welche sich direkt belegen oder indirekt rekonstruieren lassen und welche die sukzessive kognitive Entwicklung innerhalb der Gattung des Menschen dokumentieren, so behandeln drittens die Sprachwissenschaften den konkreten, in Frage stehenden Forschungsgegenstand der menschlichen Sprache und stellen somit die letzte fachliche Perspektive, welche innerhalb des vorliegenden Unterfangens fundierend und mit konkreter Relevanz für die Fragestellung des Sprachursprungs eingeführt werden soll, dar. Eine primäre Begründung der vorliegenden Einführung findet sich trivialerweise im Forschungsgegenstand selbst, denn freilich ist ein Verständnis zur Konstitution natürlicher Sprachen vonnöten, wenn die Entwicklung von einem zum Erwerb von Sprache nicht befähigten Taxon hin zu einem der Sprache mächtigen Lebewesen erschlossen und verstanden werden soll. Doch auch jenseits dieser banalen Feststellung finden sich Argumente zugunsten der Sinnhaftigkeit dieses einführenden Kapitels einerseits innerhalb der Ausführungen des dem vorliegenden Kapitels vorangestellten Zitats Bickertons und andererseits darin, dass auch mit dem Begriff Sprache - ähnlich anderen in vorigen Unterkapiteln vorgestellten und erschlossenen Begrifflichkeiten - im Rahmen des populären Gebrauchs eine verfälschende bzw. problematische Auffassung zum Begriff besteht. 
So existiert wie bei Evolution auch hinsichtlich Sprache ein bei näherer Betrachtung beinahe unbrauchbar vager populärer Begriff parallel zum strengeren wissenschaftlichen Konzept. Der einschlägige Klärungsbedarf ist darüber hinaus als in besonderem Maße umfassend einzuschätzen, da jenseits jener populären begrifflichen Missverständnisse, welche die Evolutionsbiologie und die Paläoanthropologie prinzipiell ebenso wie die Linguistik aufweisen, auch innerhalb einschlägiger Kreise des Forschungsfeldes der Sprachursprungsforschung bis in die jüngere Forschung defiziente sowie defizitäre Ansichten zum Phänomen menschlicher Sprache bzw. Sprachen weiterbestehen.

In diesem Sinne soll im Nachfolgenden zunächst die naive Auffassung des populären Begriffs der Sprache korrigiert werden, bevor schließlich die strukturelle und funktionale Komplexität natürlicher Sprachen für linguistisch nicht vorgebildete Leser skizziert werden soll, um auch in dieser Hinsicht einen treffenden, wenn auch wiederum lediglich skizzierten und mithin unvollständigen, Verständnishintergrund zu bieten (für einschlägige Einführungen zum Feld und zu Schnittstellen der Sprachwissenschaften, d.h. genauer aus Sicht der germanistischen Linguistik, vgl. u.a. Busch \& Stenschke 2008, Meibauer et al. 2007 sowie Steinbach et al. 2007, sowie exemplarisch für thematisch weiterführende Einführungen bzw. Übersichten in Teilgebiete der Linguistik Hall 2000, Eroms 2000, Meibauer 2008, Engel 2009 und Burger 2010). Zugunsten einer adäquaten Veranschaulichung des zu Zeigenden wird kontrastiv auf die kommunikative Befähigung nicht-menschlicher Tiere eingegangen, bevor sprachliche Dimensionen jenseits jener nicht-menschlichen Kommunikation in knapper Weise aufgezeigt werden sollen. Das vorliegende Kapitel kulminiert schließlich in einem ebenso knappen Ausblick auf die Rolle der Linguistik in der Rekonstruktion der Phylogenese von Sprache.

\subsection{Sprache, Kommunikation und die Biologie von Sprache}

Einen Einstieg bietet wie eingangs angemerkt die Feststellung, dass Sprache einen im populären Kontext überaus inflationär verwendeten und dabei gegenüber dem linguistischen Konzept meist verfälschenden Begriff darstellt. Die Tragweite dieser Beobachtung spannt von populärwissenschaftlichen Formulierungen wie der Sprache der Bienen (vgl. für ein Beispiel aus der populären Berichterstattung Knauer 2011), angefeuert durch die fachliche Bezeichnung des Kommunikationssystems der Honigbiene als Tanzsprache bzw. „dance language“ (bspw. von Frisch 1965 und Dyer 2002), sowie Konzepten wie der Körpersprache bis zu Begriffen wie der Sprache(n) der Liebe in prominenten Formen der Paartherapie (Chapman 2003) sowie die Sprache der Depression hinsicht- 
lich potenzieller Ansätze diagnostischer Früherkennung innerhalb Verhaltensmustern im Frühstadium klinisch relevanter Depression (bspw. Zink 2018 nach Al-Mosaiwi \& Johnstone 2018) - und mitunter sind es auch prominente Sprachursprungsforscher, welche beispielsweise systematische und dabei teils hierarchische Regularitäten in Vogelgesang erkennen und in Folge von einer „Vogelsyntax“ sprechen, um dann klärend anmerken zu müssen, dass hierbei keine mit dem Menschen vergleichbare Befähigung zu syntaktischen Strukturen gemeint sei (vgl. auch die Perspektiven von Berwick et al. 2011 und 2012, Mol et al. 2017, Prather et al. 2017 sowie Bolhuis et al. 2018a und 2018b).

Bickerton (2007: 518) stellte bereits im Sinne der vorliegenden Worte fest, „[that] ,phonological syntax ${ }^{\text {‘ }}$ or ,the syntax of birdsong ${ }^{6}$ [...] are mere metaphors“ und dass die menschliche Befähigung zu Syntax hinsichtlich Struktur sowie Funktion klar einzigartig ist - ein Umstand, der gemeinhin von linguistisch nicht oder wenig vorgebildeten Wissenschaftlern hoffnungslos unterschätzt wird. Stattdessen wird das Phänomen der menschlichen Sprache konzeptuell oftmals wie eine Blackbox, d.h. als einen bloßen Platzhalter, behandelt, deren interner Aufbau nicht näher untersucht oder verstanden werden muss bzw. deren evolutionäre Entwicklung durch nicht-linguistische Perspektiven erschöpfend ergründet werden kann - und kommunikative Fähigkeiten nicht-menschlicher Tiere sind damit axiomatisch adäquate Vorläufer menschlicher Sprache (vgl. bspw. Herangehensweisen wie bei Corballis 2002 und Tomasello 2008).

Oftmals fällt es innerhalb entsprechender Veröffentlichungen der populären Berichterstattung sowie nicht-linguistischer Disziplinen schwer, kontextsensitiv zu entscheiden, ob entsprechende Aussagen bzw. Meinungen von den Äußernden mit fahrlässigem Desinteresse oder mit naiver Ernsthaftigkeit vorgebracht werden. So oder so - also unabhängig davon, ob intendiert oder ungewollt - ist jedoch eine fehlleitende Lesart, welche Sprache einem bloßen Kommunikationssystem (oder gar Kommunikation ganz allgemein) gleichsetzt, $\mathrm{zu}$ diagnostizieren. Fehlleitend ist eine derartige Gleichsetzung aufgrund des Umstands, dass die Komplexität, Leistungsfähigkeit und Anwendung menschlicher Sprache teils in quantitativer und teils in qualitativer Hinsicht von nichtmenschlicher Kommunikation zu unterscheiden ist. Ähnlich wie bei der PuzzleMetapher gegenüber der Paläontologie gilt also auch hier höchstens artifiziell Vergleichbarkeit bei tatsächlich qualitativer Unvergleichbarkeit.

Die Reichweite dieser Feststellung beginnt bei den eine Sprachbefähigung ermöglichenden biologischen Fundamenten, die innerhalb der humanspezifischen Evolution teilweise eine einzigartige Form eingenommen haben, schließt Sprache unterliegende sowie mit Sprache interagierende kognitive Domänen, 
welche im Menschen eine besondere Leistungsfähigkeit bzw. eine besonders ausgeprägte reziproke Integration erreicht haben, mit ein und endet bei der hochkomplexen, multidimensionalen formalen Struktur natürlicher Sprachen einschließlich der wenigstens ebenso umfassend sowie vielseitig ausgeprägten funktionalen Leistungsfähigkeit sprachlicher Systeme - unzweifelhaft ein Einzelfall der Tierwelt. Überdies sind die beiden Phänomene der Kommunikation und der Sprache bereits substanziell voneinander zu unterscheiden, auch wenn nach einschlägigen Definitionen eine gewisse Unschärfe verbleibt.

Es kann nicht verneint werden, dass innerhalb der Alltagserfahrung der Eindruck entsteht, Sprache sei schlicht ein besonders leistungsfähiges und systematisches Kommunikationssystem - und in der Tat wird Sprache beständig kommunikativ genutzt. Zugleich ist ebensowenig bestreitbar, dass im Verlauf des Erstspracherwerbs eine sukzessiv fortschreitende Transformation der kognitiven Welt des jeweiligen Individuums stattfindet und dass ebendiese Transformation ausbleibt bzw. als unvollendet $\mathrm{zu}$ sehen ist, wenn kein Erstspracherwerb stattfindet. In diesem Sinne ist Sprache zwar ein Kommunikationssystem, jedoch nicht ausschließlich ein Kommunikationssystem, sondern zugleich auch ein kognitiv strukturierendes Mittel, welches wenigstens graduell gleich den beiden Hälften eines Reißverschlusses mit manchen höheren kognitiven Prozessen, Befähigungen und Gedankenkonstrukten verzahnt $\mathrm{zu}$ sein scheint (vgl. bspw. de Villiers 2007, Winawer et al. 2007, Lupyan 2012, de Villiers \& de Villiers 2014 sowie Thierry 2016). In ähnlicher Weise umfasst der Begriff der Kommunikation sowohl sprachliche Kommunikation als auch Gestik, Mimik, Lachen oder Weinen, Berührungen und weitere Phänomene, welche nicht der sprachlichen Domäne im engeren, technischen Sinn angehören. Die beiden Konzepte überlappen damit und bilden eine Schnittmenge, aber fordern aufgrund des nur teilweise gemeinsam abgedeckten Bereichs eine jeweils eigene und voneinander abzugrenzende Beschreibung.

Die soeben gemachte Feststellung einer teilweisen biologischen sowie strukturellen Einzigartigkeit soll freilich nicht missverstanden werden. Eine Vielzahl von Organismen hat auf die eine oder andere Weise Einzigartigkeit hervorgebracht, um ihren jeweiligen ökologischen Nischen durch ihre konkreten Innovationen angemessen zu entsprechen. Kein Landlebewesen erreicht im Sprint die Spitzengeschwindigkeit eines Geparden, keine andere Art erreicht die Größe und Masse eines Blauwals und kein anderes Lebewesen zeigt eine mit dem Menschen vergleichbare Befähigung zum Erlernen natürlicher Sprachen. Objektiv und evolutionsbiologisch gesehen besteht zwischen allen diesen Feststellungen kein erkennbarer Unterschied. Weiterhin war der Weg zur modernen Sprachbefähigung und damit zum Phänomen natürlicher Sprachen - wie in der 
evolutionsbiologischen Einführung auch bereits angemerkt - im Sinne der Evolutionstheorie wenigstens hinsichtlich den biologischen Grundlagen ein gezwungenermaßen sukzessiver Prozess und konstruiert sich damit auf Basis der in vormenschlichen Lebensformen bereits vorhandenen Physiologie.

Doch auch im Kontext dieser Aussagen zeigen sich die jeweiligen Start- und Endpunkte dieser phylogenetischen Kontinuität als qualitativ nicht vergleichbar. Beide Phänomene - d.h. einerseits der Ausgangspunkt nicht-menschlicher Kommunikation und andererseits der Endpunkt menschlicher Sprache - existieren heute parallel und können damit synchron verglichen werden, wobei sich ebenjene qualitative Diskontinuität offenbart. Aus dieser Diskontinuität folgt schließlich auch die Feststellung, dass jenes vormals festgestellte Vorgehen, axiomatisch Vergleichbarkeit zwischen nicht-menschlicher Kommunikation und menschlicher Sprache zu unterstellen, einem adäquaten Verständnis der tatsächlichen Situation abträglich ist. In diesem Sinne bedarf es für eine angemessene wissenschaftliche Ergründung der Entwicklung hin zum Phänomen der menschlichen Sprache einer linguistisch informierten Perspektive. Es verbleibt, in ebendiesem Sinne im Folgenden die Diskontinuität zwischen nichtmenschlicher Kommunikation und menschlicher Sprache $\mathrm{zu}$ verdeutlichen. In diesem Vorgehen ist möglich, sowohl eine Skizze nicht-menschlicher Fähigkeiten aufzumachen als auch einen dazu kontrastiven, knappen Überblick zum Phänomen menschlicher Sprache zu bieten. Eine Veranschaulichung hierzu soll mittels einer Analogie zu einem in geringerem Maße strittigen Forschungsbereich gegeben werden. Dieses Vorgehen soll es ermöglichen, sowohl die quantitativen Unterschiede zwischen nicht-menschlicher Kommunikation und menschlicher Sprache offenzulegen als auch ferner die Natur der qualitativen Kluft eingängig zu erleuchten.

\subsection{Eine biologisch-kulturelle Analogie zwischen Mathematik und Sprache}

Eine Vielzahl von Tieren besitzt einen angeborenen, intuitiven Zahlen- bzw. Mengensinn, welcher bei natürlichen Verhaltensmustern wie der Nahrungsbeschaffung, innerhalb des Sozialverhaltens sowie auch im Kontext mancher Gefahrensituationen von immenser Bedeutung ist und daher äußerst umfassend im Tierreich etabliert und erhalten wurde - den Menschen eingeschlossen. ${ }^{28}$

28 Für Beispiele zum nicht-menschlichen Zahlensinn siehe von Schimpansen (Woodruff \& Premack 1981) über Rhesusaffen (Brannon \& Terrace 1998 sowie Drucker, Rossa \& Brannon 
Diese Befähigung impliziert jedoch weder ein abstraktes Verständnis zu den explizit ordinalskalierten Numeralen des Rechnens bzw. der Mathematik noch die kognitive Befähigung, die logische Beziehung zwischen derartigen Numeralen bzw. Operationen zu durchschauen, und ist auch hinsichtlich des quantitativen Umfangs stark begrenzt. Je nach Tierart können typischerweise drei bis fünf singuläre Elemente sowie Mengenverhältnisse mit einer Genauigkeit zwischen 2:3 und 4:5 effizient erkannt werden, auch wenn diese Beschränkung innerhalb einiger Arten durch vage Einschätzungen wie „wenige“ und „viele“ eine Erweiterung findet und obgleich dabei - ebenfalls innerhalb ausgewählter Arten - durchaus eine nennenswerte Leistungsfähigkeit beim Vergleichen von Mengenverhältnissen bis jenseits von 20 Elementen gegeben ist (vgl. nochmals die in der vorhergehenden Fußnote genannten Studien).

In anderen Worten: Bereits die mathematische Menge der natürlichen Zahlen übersteigt ein biologisch begründetes, intuitives Verständnis von Zahlen bzw. Mengen sowohl in quantitativer als auch in qualitativer Hinsicht in bedeutsamer Weise - und das, bevor die Arithmetik, algebraische Operationen oder erweiterte Zahlbereiche berücksichtigt wurden. Dies gilt, obgleich ein bloßes Rechnen und das Arbeiten mit einfachen Mengenverhältnissen sicherlich nicht vergleichbar mit der Disziplin der Mathematik ist - denn die Geometrie, die Stochastik sowie weitere Arbeitsfelder verbleiben dabei noch völlig unbeachtet. In diesem Sinne ist der festgestellte, primär genetisch fundierte Zahlenbzw. Mengensinn, der auch außerhalb des Menschen umfassend existiert, weder quantitativ noch qualitativ mit der menschlichen mathematischen Befähigung zu vergleichen.

Dieses Bild spiegelt sich in der Komplexität natürlicher Sprachen gegenüber den kommunikativen Befähigungen nicht-menschlicher Tiere wider. Die Analogie ist nicht perfekt, denn in der Tat scheint die Mathematik - mehr noch als Sprache - kein streng evolutionäres, sondern ein primär kulturelles, weitestgehend kognitiv begründetes Phänomen zu sein. Dies zeigt sich teils in der klaren Abhängigkeit von kultureller Tradierung hinsichtlich Zählen, Rechnen und

2016) zu Bären (Vonk \& Beran 2012), Tauben (Scarf, Hayne \& Colombo 2011), Haushühnern (Rugani, Vallortigara \& Lucia 2015), Fischen (Agrillo et al. 2008 sowie 2009, Petrazzini et al. 2016), Tintenfischen (Huang et al. 2019), Salamandern (Uller et al. 2003) und selbst Insekten (Dacke \& Scrinivasan 2008, Gross et al. 2009, Howard et al. 2018) sowie Spinnentieren (Nelson \& Jackson 2012). Auch der Mensch besitzt einen solchen untergründigen, intuitiven Zahlensinn, welcher spontane Schätzungen im Bereich niedriger Numerale sehr effizient macht, jedoch jenseits davon unzuverlässig wird (vgl. von der historisch frühen Veröffentlichung Jevons 1871 bis zur jüngeren Forschung, zu indigenen Völkern wie bei Dehaene et al. 2008 und zu Vergleichen zwischen Menschen und nicht-menschlichen Tieren wie bei Agrillo et al. 2012). 
allgemeiner der Mathematik (bspw. Dehaene et al. 2008 und Spaepen et al. 2012) und teils auch darin, dass viele mathematische Konzepte sowie die gesamte höhere Mathematik erst in historischen Zeiträumen eingeführt und integriert wurden - so beispielsweise das heute ubiquitäre Konzept einer expliziten Null (Kaplan 2000).

Dies gilt, obgleich die menschliche mathematische Befähigung sicherlich durch eine identifizierbare und klar verortete neuroanatomische Infrastruktur begründet wird und damit auch einen fixierten genetischen Hintergrund impliziert. In der Tat scheint die menschliche neuroanatomische Infrastruktur mit dem bereits skizzierten, in der Tierwelt weitläufig vorhandenen inhärenten Zahlensinn in enger Verbindung zu stehen und nicht näher mit sprachlichen lokalen oder funktionalen Zentren zu korrelieren (vgl. Amalric \& Dehaene 2008), was sich auch innerhalb der Akalkulie, d.h. der erworbenen Unfähigkeit zu mathematischer Kalkulation, zeigt, da auch hier eine enge Korrelation mit sprachlicher Befähigung nicht erkennbar ist (Cohen et al. 2008 sowie die dort zusammengefassten Studien), auch wenn freilich für ein Verbalisieren von Numeralen keiner der beiden Komplexe umfangreich beeinträchtigt sein darf.

Die menschliche Sprachbefähigung ist dagegen vergleichsweise umfangreich durch biologische Anpassungen zu begründen. Änderungen beinhalten hier beispielsweise das Innenohr, den Kehlkopf, den Mund- und Rachenraum, eine erstaunlich umfassende und effiziente Kontrolle über die Atmung und eine wenigstens teilweise Modifikation der involvierten neuroanatomischen Strukturen. Diesen Feststellungen entsteht auch keine kritische Minderung durch die Anmerkung, dass viele dieser biologischen Anpassungen anscheinend die Effizienz oraler sprachlicher Kommunikation mehr denn unmittelbar die unterliegende linguistische Befähigung betreffen.

Außerdem verbleibt auch im vorliegenden Kontext die aufgemachte Analogie eine sinnvolle: Einerseits existiert eine zur mathematischen Befähigung im Groben vergleichbare Diskontinuität zwischen voll ausgebildeter menschlicher Sprache und nicht-menschlicher Kommunikation trotz im Tierreich durchaus vorhandener archaischer biologischer Grundlagen und andererseits besteht durchaus ein demonstrativer Mehrwert im plausiblen Aufzeigen existierender qualitativer Kluften zwischen der nicht-menschlichen und der menschlichen Seite. Mithin ist sowohl hinsichtlich der Mathematik als auch hinsichtlich Sprache eine gewisse evolutionsbiologische Kontinuität festzustellen und zugleich eine qualitative Kluft zwischen der nicht-menschlichen und der menschlichen Seite zu bemerken. In diesem Sinne seien jene zwei vorangegangenen Absätze, welche die Skizze nicht-menschlicher mathematischer Befähigungen beinhal- 
ten, nochmals aufgegriffen und mit Bezug auf die Thematik kommunikativer bzw. sprachlicher Fähigkeiten angepasst:

Eine Vielzahl von Tieren besitzt die angeborene, intuitive Befähigung zur Produktion kommunikativer Signale und zugleich die Fähigkeit, ebensolche kommunikative Signale anderer Individuen spontan $\mathrm{zu}$ interpretieren. Diese Faktoren sind bei natürlichen Verhaltensmustern wie der Nahrungsbeschaffung, innerhalb des Sozialverhaltens sowie auch im Kontext mancher Gefahrensituationen von immenser Bedeutung und sind daher äußerst umfassend im Tierreich etabliert und erhalten - den Menschen eingeschlossen. ${ }^{29}$ Diese Befähigung impliziert jedoch weder ein abstraktes Verständnis zu semantischen Kategorien noch die kognitive Befähigung, die logischen Beziehungen paradigmatischer und syntagmatischer Relationen innerhalb strukturierter Äußerungen zu durchschauen, und ist auch hinsichtlich des quantitativen Umfangs sowie der Produktionseffizienz einschließlich der Flexibilität stark begrenzt. Je nach Tierart kann die Zahl der kommunikativen Elemente stark variieren, ist jedoch in jedem Fall limitiert auf eine beschränkte Anzahl vager bzw. gegebenenfalls näherungsweise leerer, meist imperativer und emotiver kommunikativer Einheiten, auch wenn diese Beschränkung in einigen Arten durch eine begrenzte Flexibilität innerhalb der Produktion dieser kommunikativen Einheiten sowie mithilfe der reichen kognitiven Welt der jeweiligen Rezipienten eine Erweiterung findet und obgleich dabei - ebenfalls innerhalb ausgewählter Arten - eine nennenswerte Leistungsfähigkeit beim Übermitteln von basalen Informationen sowie bei der Koordination sozialer Interaktionen gegeben ist.

In anderen Worten: Bereits die Sprachbefähigung von Kleinkindern übersteigt ein biologisch begründetes, intuitives kommunikatives Verständnis sowohl in quantitativer als auch in qualitativer Hinsicht in bedeutsamer Weise und das, bevor die typischerweise diffizile Phonologie sowie Morphosyntax menschlicher Sprachen oder der erstaunliche Umfang eines natursprachlichen Lexikons Berücksichtigung finden. Dies gilt, obgleich diese klassische Kombination der Semantik mit der Morphosyntax in einem strukturellen Sinn nebst der Phonologie sicherlich nicht deckungsgleich mit der Disziplin der Linguistik ist - denn die funktionale Seite einschließlich grammatischer Kategorien, die Pragmatik sowie weitere Arbeitsfelder verbleiben dabei noch völlig unbeachtet. In diesem Sinne ist die festgestellte, primär genetisch fundierte kommunikative Befähigung, die auch außerhalb des Menschen umfassend attestiert werden

29 Nicht-menschliche Kommunikation wird umfangreich beforscht. Entsprechende Studien sind an späterer Stelle mehrfach von Interesse in der jeweiligen Diskussion und sollen daher an gegebener Stelle noch ausgenommen verbleiben. 
kann, weder quantitativ noch qualitativ mit der menschlichen sprachlichen Befähigung zu vergleichen.

Zusammenfassend kann festgestellt werden, dass mit der Beschreibung nicht-menschlicher kommunikativer Fähigkeiten sowie korrelierender biologischer Grundlagen innerhalb der nicht-menschlichen Tierwelt weder Analogien mit Sprache und Sprachbefähigung aufgemacht werden können noch eine ausreichende Ergründung der phylogenetischen Entwicklung derselben vorgelegt werden kann, auch wenn sich innerhalb der Literatur vielerorts freizügige Verwendungen sprachwissenschaftlicher Begrifflichkeiten - wie bereits beispielhaft angeführt derjenige der Syntax - finden und obwohl derartige nichtmenschliche Strukturen ähnlich der Situation des intuitiven Zahlen- bzw. Mengensinns gegenüber der Mathematik durchaus eine mehr oder minder weitreichende biologische oder strukturelle Grundlage im Aufbau hin zu ausgebildeter Sprache bieten. Dies gilt sowohl in phylogenetischer Hinsicht, d.h. außerhalb des Menschen, als auch in ontogenetischer Hinsicht, also im Kontext des menschlichen Erstspracherwerbs.

\subsection{Zur Natur der Phylogenese von Sprache}

Um Missverständnissen vorzubeugen sei hiermit noch explizit festzustellen, dass damit in keiner Weise die Meinung vertreten werden soll, dass die menschliche Sprachbefähigung völlig de novo entstanden sei oder gar ein metaphysisches, nicht von biologischen Grundlagen abhängiges Phänomen darstelle. Ganz im Gegensatz hierzu zeigen mehrere Klassen von Tieren durchaus Regularitäten und eine nennenswerte Leistungsfähigkeit hinsichtlich Produktion, Rezeption und struktureller Flexibilität nicht nur auf quasi-syntaktischer Ebene, sondern auch in anderen sprachlich relevanten Bereichen. Es sind also nicht zuletzt die naiv als Syntax bezeichneten Strukturen, welche den Vergleich nicht-menschlicher Kommunikation mit menschlicher Sprache nicht ohne jegliche Plausibilität motivieren, und mit Sicherheit stellt das Phänomen der Sprache einen prinzipiell materiellen, d.h. biologisch greifbaren und neuroanatomisch verortbaren, Gegenstand dar. Dies zeigt allein schon die Pathologie bekannter Sprachstörungen, zu welchen beispielhaft Aphasien zu nennen sind und welche nicht nur linguistisch, sondern auch aus den Perspektiven weiterer Disziplinen wie den Neurowissenschaften ausgiebig untersucht werden. Doch auch im Kontext dieser Klärungen zeigt sich nach wie vor die weitläufig naive und damit hinsichtlich analytischer Schärfe ungenügend genaue Herangehensweise der einschlägigen Literatur als bedenklich, denn trotz der Bedeutung der biologischen Grundlagen sowie der neuroanatomischen Infrastruktur ist ein 
Erschließen und Beschreiben des Phänomens der Sprache ohne eine adäquate Berücksichtigung der linguistischen Forschung nicht in angemessener Weise möglich.

Im Sinne der bisherigen Erläuterungen sowie als Übergang zur kontrastiven Skizze sowohl nicht-menschlicher Kommunikation als auch menschlicher Sprache sei an gegebener Stelle nochmals knapp anerkannt, dass nicht-menschliche Tiere innerhalb ihrer Kommunikation weitreichend Struktur aufweisen - systematische Mehrgliedrigkeit und Modulation ihrer Rufe, Töne oder Gesänge und mitunter Kombinationen von ebensolchen - und hinsichtlich ebendieser Strukturen umfassend erforscht werden (mitunter im Kontrast mit Mechanismen menschlicher Lernfähigkeit und Sprachproduktion; vgl. neben Vorgenannten bspw. Suzuki, Wheatcroft \& Griesser 2016, Seyfarth \& Cheney 2016a und 2016b, Boë et al. 2017, Fehér et al. 2017, Prather, Okanoya \& Bolhuis 2017). Im Folgenden sei in einem ersten Schritt aufzuzeigen, in welchem Umfang eine kommunikative Befähigung innerhalb nicht-menschlicher Tiere $\mathrm{zu}$ konstatieren ist. Dabei ist auch auf die Adäquatheit der verwendeten Terminologie einzugehen. In einem zweiten Schritt soll weiterführend skizziert werden, welche Strukturen und insbesondere auch Funktionen jenseits nicht-menschlicher Kommunikation im Repertoire natürlicher Sprachen identifiziert werden können. Ein Aufzeigen der Komplexität natürlicher Sprachen soll einerseits verdeutlichen, was im Rahmen der Analogie mit der Mathematik zuvor festgestellt wurde, und andererseits im Sinne des vorigen Absatzes skizzenhaft vermitteln, in welchem Umfang die einschlägige Forschung die Verantwortung trägt, eine Analyse zum Aufkommen menschlicher Sprache sowie Sprachbefähigung unter Einbeziehung der linguistischen Perspektive zu erarbeiten und letztendlich in das Gesamtbild zu inkorporieren - wenigstens dann, wenn das Aufkommen menschlicher Sprache bzw. Sprachbefähigung adäquat erschlossen werden soll.

\subsection{Zur Verwendung linguistischer Terminologie in der interdisziplinären Forschung}

Zunächst sei zugunsten des vorliegenden Programms aufzuzeigen, dass zwischen Elementen sprachlicher Strukturen und Elementen nicht-menschlicher Kommunikation keine direkte Äquivalenz besteht. In diesem Sinne kann die linguistische Terminologie nicht naiv übertragen werden, ohne potenziell Missverständnisse hervorzubringen bzw. zu fördern - wie anhand von Syntax vormals bereits angedeutet wurde. Dennoch soll im Folgenden notwendigerweise der eine oder andere Vergleich gezogen werden, denn ohne derartige Vergleiche ist eine Diskussion letztendlich nicht möglich. Freilich geschieht dies immer in 
dem Bewusstsein, dass eben keine direkte Vergleichbarkeit besteht und nichtmenschliche Strukturen bestenfalls Proto-Varianten menschlicher Strukturen darstellen - mit nicht nur rein quantitativen, sondern auch qualitativen Unterschieden.

Recherche innerhalb der einschlägigen Literatur fördert Beispiele für die genannte Äquivalenz-Problematik: Nicht-menschliche Kommunikation beinhaltet unzweifelhaft voneinander trennbare kommunikative Einheiten, welche jedoch oftmals in zweifelhafter Weise naiv mit Wörtern oder holophrastischen Propositionen gleichgesetzt werden - und dies geschieht auch dann noch in zweifelhafter Weise, wenn darauf hingewiesen wird, dass in manchen Arten darüber hinaus die Möglichkeit der Kompositionalität zwischen solchen „Wörtern“ bzw. „Propositionen“ besteht (vgl. neben Vorgenannten Zuberbühler 2002 und 2018, Quattara, Lemasson \& Zuberbühler 2009, Arnold \& Zuberbühler 2012, Suzuki, Wheatcroft \& Griesser 2017 und 2018 sowie Townsend et al. 2018). Des Weiteren unterliegen diese kommunikativen Signale teilweise klaren Regeln bzw. klar erkennbaren Regularitäten hinsichtlich jener Kombinationen und schaffen auf diesem Wege mitunter neuartige Bedeutungen innerhalb dieser Ruf-Kombinationen, weswegen wiederkehrend eine Verbindung zum sprachlichen Phänomen der Wortstellung und damit zur Evolution von Syntax hergestellt wird.

Entgegen dieser oftmals durch Autoren der einschlägigen Forschung vorgenommenen Gleichsetzung jener fixierten „Wort“-Stellung innerhalb nichtmenschlicher Kommunikation mit (Proto-)Syntax weisen Linguisten (bspw. Bickerton 2003, Abschnitt 6, sowie 2007: 518-519) rechtens darauf hin, dass es sich hierbei um einen fehlgeleiteten sowie verfälschenden Vergleich handelt, und ebenso können jene Rufe bzw. Rufkombinationen nicht schlicht mit Wörtern oder auch Propositionen gleichgesetzt werden - d.h. das Vokabular zu sprachlichen Phänomenen lässt sich wie erwähnt nicht in unproblematischer Weise auf nicht-menschliche Kommunikation übertragen. Nachfolgend sollen diese beiden Punkte in umgekehrter Reihenfolge erläutert werden: Zunächst stehen Holophrasen, Wörter sowie kommunikative Intention im Vordergrund und anschließend wird eine Diskussion zur Wortstellung und (Proto-)Syntax zu führen sein. Im Anschluss hieran wird das Feld der nicht-menschlichen Kommunikation verlassen, um auf Strukturen und funktionale Aspekte natürlicher Sprachen eingehen zu können.

Damit im Rahmen dieses Vorhabens im direkt Folgenden keine terminologische Verwirrung aufkommt, sei knapp und daher vage angemerkt, dass Holophrasen Äußerungen darstellen, welche eine komplexe Aussage mit einem einzelnen Wort ausdrücken. Eine Proposition bezeichnet den mehr oder weni- 
ger abstrakten Inhalt eines Satzes unabhängig von seiner konkreten Form, sodass sowohl ein komplexer Satz als auch eine Holophrase in der Lage ist, den selben Inhalt, d.h. die selbe Proposition, auszudrücken. ${ }^{30}$ Propositionen - beispielsweise im Gegensatz zu Fragen - besitzen daneben einen Wahrheitswert. Der Imperativ ist schließlich Teil der grammatischen Kategorie des Modus und umfasst sprachliche bzw. kommunikative Akte im Sinne von Aufforderungen, Befehlen sowie vergleichbaren kommunikativen Handlungen.

\subsection{Zum Begriff der Holophrase in der einschlägigen Forschung}

Im Allgemeinen wird der Aussage zuzustimmen sein, dass ein reflexartig hervorgebrachtes „Whaaaa!“ beim überraschenden Zusammentreffen mit einer Spinne nicht selbstverständlich als Holophrase interpretiert werden sollte, solange Holophrasen als solche Lautäußerungen $\mathrm{zu}$ sehen sind, welche eine kommunikative Intention beinhalten oder ähnliche Kriterien der gerichteten Kommunikation erfüllen. Im Gegensatz zu dieser Anforderung stellt jenes Beispiel einen völlig unfreiwilligen Ton ohne einen vom Äußernden intendierten kommunikativen Mehrwert dar - und durchaus scheint es sich mit diversen Warn- oder Futterrufen nicht-menschlicher Tiere ebenso zu verhalten. ${ }^{31}$ In der Tat stellen viele innerhalb vorgenannter Studien diskutierte Rufe nichtmenschlicher Tiere reflexartige Äußerungen dar und sind daher mit geringer oder keiner kommunikativer Intention versehen (vgl. bspw. Fischer \& Price 2017). ${ }^{32}$ Damit entbehren diese Elemente ganz oder weitestgehend einer Reflexi-

30 So ist der propositionale Inhalt der beiden Äußerungen „Da sind Kühe im Garten!“ und „Kühe!“ einer überraschten Person beim Blick in den Garten trotz der abweichenden Form als gleichwertig anzusehen.

31 Freilich ist denkbar, dass an Stelle des vormals genannten „Whaaaa!“ ein reflexartiges „Ach du meine Güte!“ vorgebracht wird. Auch dieses entbehrt als Äußerung anscheinend jeglicher kommunikativen Intention und besitzt dennoch eine linguistisch komplexe Form. Obwohl es auf den ersten Blick so erscheinen mag, ist damit jedoch die vorliegende Diskussion nicht zu umgehen. Erst der Erwerb von Sprache ermöglicht den späteren reflexartigen Abruf sprachlicher Elemente. Im gegebenen Argument zu nicht-sprachlichen holophrastischen Rufen kann bzw. muss daher weiterhin im Sinne nichtsprachlicher reflexartiger Ausrufe argumentiert werden.

32 Dem ist nicht zu folgern, dass hinsichtlich ebensolcher Rufe nicht-menschlicher Tiere keinerlei Flexibilität besteht. Durchaus können Rufe taktisch unterdrückt oder übersteigert werden, um konkreten Situationen adäquat zu begegnen (vgl. neben andernorts Genannten auch Silk et al. 2016 sowie Cheney \& Seyfarth 2018). Dennoch befinden sich viele solcher Rufe 
on über den kommunikativen Akt sowie zur jeweiligen kommunikativen Bedeutung, auch wenn sie nichtsdestotrotz eine stabile, klare Form besitzen und aufgrund der Korrelation mit einem konkreten Kontext aus Sicht des Rezipienten eine inhärente Bedeutung tragen, womit eine interpretierbare und damit passiv kommunikative Äußerung vorliegt.

Da jedoch bereits frühkindliche Holophrasen - unabhängig davon, ob es sich um eine propositionale oder eine imperative Äußerung handelt - im Allgemeinen einen gerichteten sowie intentionalen kommunikativen Akt des Produzenten implizieren, kann eine direkte Vergleichbarkeit hier nicht konstatiert werden (vgl. bspw. die Einführungen in den Kindspracherwerb von KlannDelius 2008, Kauschke 2012 sowie Szagun 2013). In anderen Worten: Die vormals genannte und in der Literatur verbreitete, nachteilig vage Definition einer Holophrase als eine komplexe Aussage, welche mittels einer simplen Form zum Ausdruck gebracht wird, erlaubt für die Bezeichnung von einem großen Umfang nicht-menschlicher Rufe als Holophrasen, während bereits Kleinkinder eine in kommunikativer Hinsicht substanziell abweichende Form der Holophrase verwenden.

Zur Verdeutlichung solcher frühkindlicher Holophrasen sind entsprechende Beispiele vorzubringen. Dabei kann die nachfolgende Äußerung (1a) zwar als das konkrete Wort Ball aufgefasst werden, jedoch bestehen ferner eine Reihe von Lesarten im Sinne einer Holophrase mit den Bedeutungen (1b) bis (1e) nebst weiteren möglichen, hier jedoch nicht gelisteten Bedeutungsvarianten:
a. Ball!
b. Da ist ein Ball!
c. Gib mir den Ball!
d. Ich möchte einen Ball!
e. Geh zum Ball!

Als zweifelhaft zeigt sich dabei die Unterstellung, dass jene erwähnten nichtmenschliche Laute - d.h. konkrete Warn- oder Futterrufe, welche beispielsweise auf die Gefahr eines Adlers oder auf den Fund von Früchten hinweisen einer derart reichen Verwendung dieses Wortes als Holophrase entsprechen. Ebenso wie Ausrufe des Erschreckens angesichts der plötzlichen Begegnung mit einer Spinne entsprechen auch jene reflexartig ausgestoßenen, ungerichteten Rufe oder Töne nicht der aufgezeigten holophrastischen Verwendung lexikali-

näher an passiven Ausrufen denn an sprachlichen Äußerungen und können damit prototypischen, d.h. sprachlichen, Holophrasen nicht entsprechen. 
scher Elemente im Spracherwerb von Kleinkindern. Soll dennoch weiterhin von Holophrasen hinsichtlich jener Art nicht-menschlicher Kommunikation gesprochen werden, so böte sich die Unterscheidung in zwei holophrastische Typen an, wobei einerseits starre, reflexartige sowie typisch ungerichtete kommunikative Einheiten von andererseits flexiblen, intentionalen sowie typisch gerichteten und potenziell eine Grundlage für die Entwicklung hin zu Protosprache stellenden Einheiten unterschieden werden können.

Freilich sind kommunikative Akte nicht-menschlicher Tiere im Kontrast zur soeben durchgeführten Diskussion nicht auf jenen passiven, ungerichteten Typus beschränkt, sondern nähern sich dem Begriff einer frühkindlichen Holophrase teilweise durchaus an. In diesem Sinne besteht insbesondere bei nichtmenschlichen Menschenaffen - aber auch bei weiteren nicht-menschlichen Tieren - ein nicht zu unterschätzendes Bewusstsein über die eigene Kommunikation. Manche Rufe bzw. auch gestische kommunikative Akte nichtmenschlicher Tiere implizieren mitunter komplexe Bedeutungsinhalte, werden mit einer eingeschränkten, jedoch eben durchaus vorhandenen, metakognitiven Intentionalität eingesetzt und beinhalten entgegen früherer Annahmen auch in der oralen Modalität eine nennenswerte Flexibilität.

Insbesondere Schimpansen zeigen sich hier äußerst leistungsfähig und erlernen genetisch nicht vorgegebene Laute (Hopkins, Tagliatatela \& Leavens 2007 und 2011), modifizieren genetisch fundierte Laute kontextsensitiv (Kalan, Mundry \& Boesch 2014, Crockford, Gruber \& Zuberbühler 2018), kombinieren Laute produktiv (Crockford \& Boesch 2005), verwenden Laute mit gerichteter Intention und bezogen auf bestimmte Individuen (Schel et al. 2013a nebst 2013b), schaffen hinsichtlich Ruf-Modifikationen kulturelle Traditionen innerhalb einer Gruppe (Watson et al. 2015), kodieren Informationen zur Identität des Produzenten eines Rufes in ebendiesem Ruf (Fedurek, Zuberbühler \& Dahl 2016) und berücksichtigen Anwesenheit nebst Identität von Zuhörern (Seyfarth \& Cheney 2012, Kalan \& Boesch 2015) bzw. berücksichtigen den Wissensstand von Gruppenmitgliedern (Crockford et al. 2012). Darüber hinaus zeigen sie gegenüber ihren oralen kommunikativen Fähigkeiten nochmals gesteigerte Produktivität und Flexibilität im Rahmen ihrer gestischen Kommunikation - entgegen populärer Vorurteile auch im Rahmen von Zeigegesten (vgl. bspw. Tomasello et al. 1994, Pollick \& de Waal 2007, Hopkins et al. 2013, Hobaiter \& Byrne 2014). Zuletzt ist eine hohe kommunikative, d.h. genauer interpretative, Leistungsfähigkeit insbesondere auch auf Empfängerseite gegeben, was die erstaunlich hohe Leistungsfähigkeit jener Kommunikation trotz weitläufiger Einschränkungen innerhalb der Flexibilität und Produktivität seitens des Senders ermöglicht (siehe u.a. Bar-On 2013, Fitch 2015 sowie Fischer \& Price 2017). 
In der hier aufgemachten kommunikativ und kognitiv komplexen Situation ist eine Vergleichbarkeit des Terms der Holophrase bei nicht-menschlichen kommunikativen Signalen mit derjenigen Verwendung im Kontext des frühen Kindspracherwerbs mit größerer Plausibilität vertretbar als es noch hinsichtlich der zuvor diskutierten Ruftypen der Fall war. Im Kontrast zu diesem Zugeständnis ist an gegebener Stelle allerdings weiterführend darauf hinzuweisen, dass der Begriff der Holophrase auch auf die Sprachverwendung von Erwachsenen angewandt werden kann und hier eine zusätzliche Erweiterung des Begriffs impliziert. In diesem Sinne stehen Holophrasen nicht wie bei Kleinkindern in Ermangelung komplexer sprachlicher Befähigung an Stelle einer ausformulierten sprachlichen Äußerung, sondern implizieren eine Ersetzung der sprachlichen Äußerung mit dem Zweck der stilistischen oder pragmatischen Funktion, und setzen damit sowohl beim Produzenten als auch beim Rezipienten eine versierte Interpretation der Holophrase als Platzhalter ebenjener komplexen Aussage voraus. Da eine derartige Verwendung bereits innerhalb der sprachlichen Entwicklung von Kleinkindern die frühere Verwendung des zuvor skizzierten simpleren Typs der Holophrase weitgehend verdrängt, ist trotz der bereits geschehenen klärenden Unterscheidung in zwei Typen der Holophrase mit angemessener Vorsicht mit dem Begriff umzugehen - ein Umstand, der freilich in der einschlägigen Forschung weitgehend keine Berücksichtigung findet und damit gemeinsam mit anderen Aspekten zu einer artifiziellen, lediglich scheinbaren Vergleichbarkeit von Sprache und nicht-menschlicher Kommunikation führt.

Zusammenfassend sind hiermit drei grobe Typen der Holophrase identifiziert und - zugegebenermaßen ohne für eine brauchbare Anwendung angemessene Begriffsbestimmungen - skizziert worden, von welchen der simpelste Typus weitläufig im nicht-menschlichen Tierreich aufgefunden werden kann, ein weiterer möglicherweise bzw. näherungsweise in wenigen Arten zu finden ist und von welchen der dritte schließlich ein strikt sprachliches Phänomen darstellt. Eine abschließende Abgrenzung zwischen diesen Typen ist hinsichtlich konkreter Fälle in Ermangelung einer direkten Einsicht in die mentale Welt von Individuen nur eingeschränkt bzw. womöglich nicht möglich, jedoch verbleibt eine derartige prototypische Unterscheidung im Sinne jener Typen oder eines vergleichbaren Ansatzes sinnvoll. Mithin ist als gewinnbringend anzusehen, eine solcherlei Ansätzen entsprechende analytische Ergründung gegebener kommunikativer Gegebenheiten bzw. Befähigungen gewissenhaft durchzuführen, anstatt bei der pauschalen und missverständlichen Anwendung des Begriffs der Holophrase als ein in einer jeden kommunikativen Situation einheitliches Phänomen zu verbleiben. 


\subsection{Sprachexperimente mit Primaten und der Begriff des Wortes}

Nun ist zugunsten der Reichweite der vorliegenden Skizze jenseits der soeben diskutierten, in der freien Natur vorkommenden und näherungsweise holophrastischen Verwendung von Signalen noch auf ein weiteres kommunikatives Phänomen knapp einzugehen: In Gefangenschaft zeigen nicht-menschliche Tiere und insbesondere nicht-menschliche Menschenaffen jenseits des eben Diskutierten die Befähigung, relativ konkrete, Wörtern vergleichsweise ähnliche Repräsentationen zu Konzepten zu erlernen - d.h. zu verstehen und anzuwenden sowie dabei zu kurzen Aussagesträngen zu kombinieren, auch wenn der Umfang dieser Kompetenzen bis heute zur Diskussion steht (vgl. als eine strenge Auswahl Gardner \& Gardner 1969, Patterson 1978, Terrace et al. 1979, SavageRumbaugh et al. 1993 sowie Beran \& Heimbauer 2015). Zuletzt sind Kombinationen kommunikativer Einheiten auch jenseits einer Modalität möglich, sodass zuvor diskutierte nicht-sprachliche Typen der Holophrase sowie ebenjene Wörtern ähnliche Einheiten mit deiktischen Gesten kombiniert werden können (Greenfield, Lyn \& Savage-Rumbaugh 2008). Während die kommunikative Leistungsfähigkeit, die hierbei im Rahmen mancher dieser Experimente erreicht wurde, durchaus beeindruckend erscheinen mag, so sind die beiden Anmerkungen zu machen, dass einerseits das dort Erreichte gegenüber den Meilensteinen eines typischen Erstspracherwerbs in jeglicher Hinsicht verblasst und dass andererseits wiederum die Äquivalenz der Terminologie in Frage steht, was nachfolgend auf mehreren Ebenen verdeutlicht werden soll.

Erstens stellen Wörter nicht die kleinsten bedeutungstragenden sprachlichen Elemente dar, sondern besitzen eine komplexe interne Struktur. Die nachfolgenden Beispiele (2a) bis (2d) suchen dies unter Verzicht einer weiterführenden linguistischen Erläuterung zu verdeutlichen. Jene Beispiele zeigen auch ohne technische Terminologie, dass selbst das unscheinbare, kurze Wort Wort aus kleineren Einheiten besteht, wobei anzumerken ist, dass diese kleineren Einheiten keinesfalls über die graphische Repräsentation mittels Buchstaben zu definieren sind, sondern über die nicht immer klar mit Buchstaben oder Buchstabenkombinationen korrelierenden Laute, d.h. Phoneme, einer Sprache. ${ }^{33}$ Ein

33 So stellt die Buchstabenfolge sch wie in Schule das Phonem [S ] dar, während derselbe Laut in Konsonantenkombinationen allein durch $s$ repräsentiert werden kann (vgl. $s p$ und $s t$ in sparen oder stehen), und sowohl „ee“ als auch „eh“ und sogar der einzelne Buchstabe „e“ sind in der Lage, den Langvokal [e:] auszudrücken (wie in Meer, mehr und Met). Schließlich steht 
Austausch dieser Einheiten mit anderen möglichen Einheiten führt $\mathrm{zu}$ völlig anderen Worten, womit das diffizile Zusammenspiel zwischen diesen beiden sprachlichen Ebenen verdeutlicht wird. Die feingliedrigere dieser beiden Ebenen findet sich nicht in vergleichbarer Weise im nicht-menschlichen Tierreich und wird auch innerhalb von Sprachexperimenten mit Menschenaffen ignoriert, denn dort sind die jeweils verwendeten, Wörtern scheinbar ähnlichen Lexeme nicht in gleicher Weise konstruiert, sondern stellen atomare, vereinfachte Einheiten dar.
a. Wort, Hort, dort, fort, Fort, Port
b. Wort, Wirt, Wert, wehrt, währt, Wart, wart, wahrt
c. Wort, wollt
d. Wort, Ort

a. Der Laufende läuft laufend den laufenden Lauf.

b. Täglich und tagelang tagt der betagte Bundestag.

Zweitens sind Wörter keine homogene Klasse von Elementen, sondern lassen sich nach jeweils gemeinsamen Merkmalen in voneinander differenzierte Wortarten einordnen - im Deutschen wie in vielen anderen Sprachen von Verben und Substantiven über eine Reihe weiterer Typen bis zu Präpositionen und Partikeln. Zwar existieren Sprachen, in denen die Wortartenunterscheidungen der klassischen Grammatiken kaum angewandt werden können, jedoch finden sich Wortarten prinzipiell in jeder Sprache. Das Deutsche bietet dabei eine vergleichbar reiche Wortbildung, wie durch die Beispiele (3a) und (3b) verdeutlicht werden soll. $\mathrm{Zu}$ beachten seien insbesondere auch voneinander abweichende Lesarten mancher Wörter, welche die Reichhaltigkeit des Prozesses zusätzlich unterstreichen, während freilich die inhaltliche Richtigkeit dieser Beispielsätze nicht von Relevanz ist. Auch hierbei besteht ein Umstand, der sich in jenen Experimenten nicht widerspiegelt, denn die dort verwendeten kommunikativen Symbole werden nicht in vergleichbarer Weise konstruiert oder verwendet.

Drittens kommen Wörter außerhalb von Holophrasen nicht als einzelne Elemente vor, sondern sind Teil komplexerer Konstruktionen, bei denen nicht zuletzt die jeweilige Wortart über die Position innerhalb dieser grammatischen Konstrukte bestimmt und bei denen nicht die freie, simple Kombinatorik man-

die graphische Repräsentation ch sowohl für [ç] in beispielsweise ich als auch für $[\mathrm{x}]$ wie in acht. 
cher nicht-menschlicher Kommunikationssysteme, sondern eine bedingte, von komplexen und mitunter hierarchischen Relationen geprägte Kombinatorik vorliegt. Das Beispiel (4a) zeigt noch zwei simple Kombinationen jeweils zweier Worte innerhalb eines Kompositums, das in struktureller Hinsicht entfernt mit erwähnter nicht-menschlicher Kombinatorik vergleichbar sein könnten. Die Beispiele (4b) bis (4i) verdeutlichen dagegen strukturelle Einschränkungen hinsichtlich Wortkombinationen innerhalb von Sätzen des Deutschen, welche sich weder auf nicht-menschliche Kommunikation generell noch auf jene experimentellen Kommunikationssysteme mit nicht-menschlichen Menschenaffen übertragen lassen. ${ }^{34}$ In der Tat implizieren Wörter im Sinne ihrer Wortarten, ihrer syntaktischen Rolle sowie ihrer Semantik die Offenheit bzw. gegebenenfalls die Notwendigkeit, weitere Worte bzw. Wortgruppen an sich zu binden und somit einen Satz produktiv zu erweitern - wenigstens theoretisch bis hin zu absurden Längen. Es verbleibt als wenig überraschend, dass auch dies jenseits vormals genannter Experimente liegt.
a. Wortfolge, Abschlussworte
b. *Hans das Haus.
c. Hans betritt das Haus.
d. `Julia verlässt ohne Bedauern.
e. Julia verlässt die Party ohne Bedauern.
f. $\quad$ Karl arbeitet gern Team.
g. Karl arbeitet gerne im Team.
h. * Luise schließt Tür.
i. Luise schließt die Tür.

Zusammenfassend implizieren die linguistischen Kategorien des Wortes respektive der Holophrase sowohl interne als auch externe Strukturen nebst kognitiver Phänomene sowie grammatischer Funktionen, welche von nicht-menschlicher Kommunikation nicht widergespiegelt werden können. Eine wichtige Erkenntnis liegt hier darin, dass es sich nicht allein um eine Problematik der quantitati-

34 Der Vollständigkeit halber ist anzumerken, dass zumindest einzelne Arten von Vögeln anscheinend in der Lage sind, einfache Einbettungen innerhalb ihres Gesangs vorzunehmen und zu fixieren. Zugleich besteht damit noch keine Vergleichbarkeit auf einem gemeinsamen Spektrum mit menschlicher Sprache - teils aufgrund der semantischen Leere von Vogelgesang und teils, weil Vögel einige Eigenschaften sprachlicher Syntax wie das Chunking zu Phrasen oder die Bildung von asymmetrischen, regierenden Abhängigkeiten nicht widerspiegeln (vgl. Vorgenannte, insbesondere Berwick et al. 2012). 
ven Leistungsfähigkeit handelt, sondern dass eine Reihe in natürlichen Sprachen vorkommender Dimensionen nicht bzw. in nur scheinbar vergleichbarer Form identifiziert werden können. In diesem Sinne kann die linguistische Terminologie sowohl hinsichtlich Holophrasen als auch mit Hinblick auf das Konzept des Wortes bestenfalls aufgrund oberflächlicher Vergleichbarkeit und in Ermangelung treffenderer Bezeichnungen herangezogen werden.

\subsection{Wortstellung und Syntax im Vergleich mit nicht- menschlichen Rufkombinationen}

Ähnlich verhält es sich mit den Feststellungen zur syntaktischen Befähigung nicht-menschlicher Tiere, welche primär auf Untersuchungen zu Regularitäten in der Rufproduktion einiger Arten - insbesondere innerhalb der Teilordnung der Affen und der Klasse der Vögel - zurückzuführen ist. Innerhalb der einschlägigen Literatur wird dabei auf die Möglichkeit der Kombination einzelner Rufe hingewiesen und hervorgehoben, dass sich solche Kombinationen als streng regulär und mitunter bedeutungstragend zeigen, um den Bezug zur Wortstellung und damit zur Syntax nahezulegen (vgl. neben Vorgenannten bspw. Hailman \& Ficken 1986, Russel \& Townsend 2017 sowie Griesser, Wheatcroft \& Suzuki 2018).

Die Diskussion zur linguistischen Perspektive zu diesem Gedankengang soll zweckmäßig knapp geschehen, um anderen Aspekten im Verlauf des vorliegenden Vorhabens ausreichend Raum zu geben. In diesem Sinne besteht der nachfolgende zentrale Diskussionspunkt darin, dass sich Untersuchungen zur Syntax natürlicher Sprachen entgegen jener Gedankengänge nur in geringem Umfang mit der horizontalen Wortreihenfolge beschäftigen. Tatsächlich zeigt sich die Wortstellung innerhalb vieler Sprachen als in unterschiedlichem Umfang flexibel und ist zwar gegebenenfalls markiert - d.h. auffällig, da von prototypischer Ordnung abweichend, und damit eine besondere Bedeutung vermittelnd - , aber eben auch nicht völlig fixiert. Dies soll mittels der nachfolgenden Beispiele 5 und 6 verdeutlicht und diskutiert werden:
a. Marie hat Peter getreten.
b. Peter hat Marie getreten.
b'. PETER hat Marie getreten.
c. Getreten hat Marie Peter.
d. ${ }^{\star}$ Hat Marie Peter getreten.
e. Hat Marie Peter getreten? 
(6)
a. Die Marie hat den Peter getreten.
b. Den Peter hat die Marie getreten.
c. Getreten hat die Marie den Peter.

Sofort wird klar, dass sich die Wortstellung des Deutschen als äußerst flexibel zeigt und damit eine Reihe von Permutationen zulässt. (5a) ist ebenso grammatisch wie (5b) sowie (5c) - und (5d) ist mit einem Punkt als Satzzeichen allein deswegen ungrammatisch, weil diese Konstruktion für die Frageform von (5e) reserviert ist. Diese Beobachtungen bleiben davon unangetastet, dass (5b) eine markierte Form darstellt, welche einer gewissen Betonung wie in (5b') bedarf, um nicht missverständlich zu sein, und typisch für konkrete Situationen - beispielsweise als Antwort auf die Frage „Wen hat Marie getreten?“ - ist. Ähnlich verhält es sich mit (5c), einer weiteren markierten und damit eine spezielle Konversationssituation implizierenden Formulierung. (6a) bis (6c) machen die durchaus grammatische Natur von (5a) bis (5c) zusätzlich ersichtlich, indem sie eine tendenziell dialektale Verwendung definiter Artikel heranziehen.

In einem genau entgegengesetzten Fall wird die horizontale Wortreihenfolge des nachfolgenden Beispiels (7a) innerhalb der Äußerung (7b) unverändert wiedergegeben, während sich zugleich die syntaktische Struktur drastisch verändert. (7a) als durchaus grammatische Äußerung, wie sie typischerweise $z u$ Beginn einer Erzählung, einer Anekdote oder eines Witzes stilistisch die gegebene Gemütslage vermittelt, sieht „Marie“ als das Subjekt des Verbs „Sagte“, dem Kopf des Satzes, vor, während (7b) „Marie“ zum Objekt eines Verbs, welches selbst wiederum dem eigentlich Kopf des Satzes untergeordnet ist und dessen Subjekt durch „Lisa“ gegeben ist, degradiert. Auch hinsichtlich Topik (Gegenstand der Aussage) sowie Agens (Handelnder innerhalb der Aussage) verschiebt sich die Aufmerksamkeit in derselben Weise, sodass ein vollständiger Bedeutungswechsel unter Einhaltung der Wortstellung eintritt. Mithin verdeutlicht dieses Beispielpaar weiter, dass die Wortstellung hinsichtlich der syntaktischen Struktur eines Satzes prinzipiell von untergeordneter Wichtigkeit ist.
a. Sagte Marie, nach München fahre sie mit dem Auto.
b. Lisa gestand, sie sagte Marie, nach München fahre sie mit dem Auto.

Entgegen dieser horizontalen Wortstellungsdiskussion beschäftigt sich die Syntaxforschung primär mit Satzgliedern - auch wenn Satzglieder bzw. auch ganze Sätze durch ein einzelnes Wort repräsentiert werden können - sowie mit hierar- 
chischen, d.h. vertikalen, Abhängigkeitsrelationen zwischen den Elementen eines Satzes. Dabei geraten unweigerlich auch grammatische Funktionen und Relationen, welche innerhalb nicht-menschlicher Kommunikation kein echtes Äquivalent zu haben scheinen, ins Blickfeld. Zur Verdeutlichung siehe Beispiel (8), welches alle Beispiele (5a) bis (5e) gleichermaßen zu repräsentieren vermag, weil sich einer derartige Darstellung mit der unterliegenden, abstrakten Struktur eines Satzes, d.h. mit den tatsächlichen Abhängigkeiten, beschäftigt, anstatt sich mit der oberflächlichen und ohnehin flexiblen horizontalen Struktur der Wortstellung zu befassen.

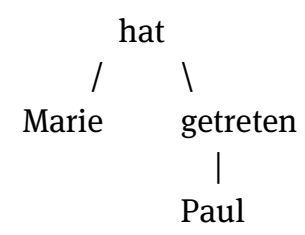

Als eine erste klärende Anmerkung ist hier noch vorzubringen, dass diese Darstellung nach gängigen dependenzgrammatischen Prinzipien, in diesem Fall konkret nach Eroms (2000), gebildet wurde und dass andere Grammatiken sowohl abweichende Dependenzgrammatiken als auch substanziell gegensätzliche Grammatiken - existieren, die gemeinhin zu anderen Stemmata bzw. Syntaxbäumen führen. Zweitens handelt es sich bei jener Abbildung zugunsten der Eingängigkeit um eine vereinfachte sowie nicht weiter erläuterte bzw. diskutierte Darstellung eines ohnehin simplen Satzes - weitere Ausführungen, gängige Nomenklatur sowie eine beispielhafte Analyse zu einem komplexen Beispiel seien an gegebener Stelle zugunsten der Eingängigkeit ausgenommen. Drittens wird wie angemerkt im vorliegenden Stemma zugunsten der Klarheit der abstrakten Abhängigkeiten nicht auf die Wortstellung des Satzes eingegangen, wobei in dem Fall, dass die Wortstellung explizit als Teil einer jeweiligen Analyse vorgesehen ist, eine entsprechende Berücksichtigung innerhalb eines Stemmas durchaus möglich wäre.

All dies soll demonstrieren, dass die Vorstellungen linguistischer Laien und damit eines nennenswerten Teils der Autorschaft innerhalb der Sprachursprungsforschung - zur Definition von Syntax bzw. zu Inhalten der Syntaxforschung die tatsächlichen Begriffe bzw. Gegenstandsbereiche nicht angemessenen abbilden. Ein gleichartiges Urteil gilt wie zuvor erläutert auch für weitere linguistische Konzepte wie diejenigen des Wortes und der Holophrase nebst Weiterem, das hier nicht erläutert werden konnte. Selbstverständlich verbleibt die Erforschung nicht-menschlicher Kommunikation trotz dieser mahnenden 
Feststellungen von kritischer Wichtigkeit in dem Unterfangen, die Evolution der menschlichen Sprachbefähigung angemessen $\mathrm{zu}$ erleuchten, und durchaus zeigt nicht-menschliche Kommunikation in vereinzelten Domänen sowie innerhalb einzelner Taxa eine nennenswerte Leistungsfähigkeit und könnte bzw. müsste die Entwicklung hin zu Sprache durchaus fundiert haben.

\subsection{Zusammenfassung des Bisherigen und zur Alleinstellung menschlicher Sprache}

Das Bisherige und damit ebenjene Punkte zusammenfassend ist festzustellen, dass nicht-menschliche Tiere sowohl hinsichtlich Form als auch hinsichtlich Funktion und insbesondere bezogen auf ihre kognitive Welt umfassend kommunizieren bzw. sich gegenseitig umfassend durch Signale beeinflussen. So zeigt die Summe der vorgenannten Studien, dass nicht-menschliche Tiere in der Lage sind, trotz vom Menschen stark abweichender physiologischer Bedingungen des Kehlkopfes sowie angeschlossener biologischer Systeme selbst feine artikulatorische Unterscheidungen vorzunehmen und ebendiese Unterscheidungen auf Seite des Rezipienten auch wahrzunehmen. Weiterhin vermitteln Tonhöhe, Lautstärke sowie Ablauf der geäußerten Laute zusätzliche Informationen zum Bedeutungsinhalt und es existiert eine rudimentäre Kombinatorik auf der Ebene der Laute bzw. Rufe. Nicht zuletzt ist eine quasi-pragmatische Referenz dank der großen kognitiven Leistungsfähigkeit seitens der Rezipienten jener Laute bzw. Rufe nennenswert ausgeprägt.

Es soll hiermit außerdem noch darauf verwiesen werden, dass weder die vorliegenden Äußerungen zur umfänglichen Inadäquatheit der aus den Sprachwissenschaften entlehnten Terminologie bei der naiven Beschreibung nicht-menschlicher kommunikativer Phänomene noch die Mahnung, dass nicht-menschliche Kommunikation nicht überinterpretiert werden sollte, singuläre Meinungen der vorliegenden Arbeit darstellen. Stattdessen werden diese Urteile freilich sowohl von Linguisten als auch von einschlägig ausreichend gebildeten Autoren geteilt. Dieser Umstand zieht sich von der Ebene der Phonologie (siehe bspw. Bowling \& Fitch 2015) über diejenige der Syntax (vgl. neben den Vorgenannten Bickerton 2003 sowie 2007 auch bspw. Fitch \& Hauser 2004 sowie Bickerton 2012) bis zu derjenigen der Pragmatik (Scott-Phillips 2010 und 2015 sowie Fitch 2015).

Das Aufzeigen der Existenz sowie die skizzenhafte Aufklärung der Natur weitläufiger Missverständnisse hinsichtlich der Vergleichbarkeit zwischen nicht-menschlicher Kommunikation mit menschlicher Sprache sei damit abgeschlossen. Zugleich konnte im Verlauf dieser Diskussion ein grober Überblick 
zur Reichweite bzw. Bedeutung nicht-menschlicher Kommunikation gegeben werden - eine für die Fragestellung der Evolution von Sprachfähigkeit gewichtige Perspektive. Freilich verblieb die Einsichtnahme in jenes vergleichende Forschungsfeld oberflächlich, womit im weiteren Verlauf der Arbeit an gegebener Stelle eine detailliertere Diskussion fortzuführen ist. Im Weiteren sei noch einmal explizit auf die Natur der Unterschiede zwischen der Kommunikation nicht-menschlicher Tiere und menschlicher Sprache einzugehen, bevor das vorliegende Kapitel durch zweierlei Gedankengänge seine Abrundung erfährt: Einerseits soll auf grammatische Funktionen natürlicher Sprachen verwiesen werden, um die besondere Leistungsfähigkeit menschlicher Sprache sowie die qualitative Kluft zur nicht-menschlichen Kommunikation zusätzlich zu verdeutlichen, und andererseits sollen konkrete Perspektiven, mit denen die linguistische Forschung die gegebene Forschungsfrage des Sprachursprungs fruchtbar informiert, knapp skizziert werden.

Hierzu ist nochmals der Gedanke aufzugreifen, dass zugunsten einer adäquaten analytischen Schärfe die Notwendigkeit besteht, die aufgemachte nichtmenschliche kommunikative Situation konzeptuell sowie gegebenenfalls terminologisch von menschlicher Sprache abzugrenzen. Wie erläutert existiert eine Problematik der Anwendbarkeit linguistischer Begrifflichkeiten schon auf scheinbar „simplen“ Strukturebenen aufgrund des Umstandes, dass sich zwar archaische bzw. rudimentäre Befähigungen bei nicht-menschlichen Tieren auf mehreren dieser Strukturebenen finden, aber dass zugleich keine direkte Vergleichbarkeit mit menschlicher Sprache gegeben ist. Dies rührt in besonderem Maße davon her, dass das Phänomen der Sprache zwei essenzielle strukturbildende Strategien impliziert, welche als in der Tierwelt einzigartig anzusehen sind. Es handelt sich dabei erstens um die systematische Integration von mehreren strukturellen Ebenen als bedeutungs- und funktionstragende Komponenten im Rahmen der jeweils hierarchisch höheren Strukturebene und zweitens um die Ausbildung abgrenzbarer struktureller sowie funktionaler Kategorien innerhalb der jeweiligen Strukturebene. Dies führt einerseits zur Ausbildung multipler struktureller sowie funktionaler Dimensionen innerhalb menschlicher Sprache, welche innerhalb der nicht-menschlichen Tierwelt nicht existieren, und führt andererseits dazu, dass vermeintlich auf die Kommunikation der nichtmenschlichen Tierwelt anwendbare Begriffe wie diejenigen des Wortes, der Holophrase oder der Syntax eben nicht ohne Verlust angewandt werden können, da innerhalb eines solchen Vorgehens die synchrone Kluft zwischen nichtmenschlicher Kommunikation und Sprache unterschlagen wird.

In anderen Worten: Im Rahmen einer phänomenologischen Betrachtung nicht-menschlicher Kommunikation sowie menschlicher Sprache, d.h. inner- 
halb einer Untersuchung der beiden Phänomene in ihrer unmittelbar gegebenen Erscheinung, ist qualitative Unvergleichbarkeit zu unterstellen. Zugleich soll nochmals betont werden, dass trotz dieser Feststellung auf evolutionsbiologischer und damit physiologischer sowie neuroanatomischer Ebene eine diachrone, phylogenetische Kontinuität postuliert werden muss, um dem wissenschaftlichen Konsens aller an der Forschungsfrage beteiligter Disziplinen gerecht $\mathrm{zu}$ werden. Hieraus ergibt sich nicht notwendigerweise die Folgerung eines inneren Widerspruchs, auch wenn diese Ausführungen dies möglicherweise auf den ersten Blick nahelegen, denn ein Ausbau nicht-menschlicher Protosysteme zu höherer quantitativer Leistungsfähigkeit - dabei eingeschlossen sei sowohl die rohe Leistungsfähigkeit einzelner Systeme als auch die Integration der Systeme ineinander sowie neuroanatomischen Innovation zur Schaffung einer strukturellen respektive funktionalen sprachlichen Infrastruktur - beinhaltet im Sinne der Überschreitung von Schwellenwerten unvorhergesehene Potenziale zu qualitativen Innovationen (vgl. die einschlägige neurowissenschaftliche Perspektive bspw. durch Sapolsky 2011 und 2017b).

\subsection{Von der Struktur zur Funktion von Sprache und zu grammatischen Kategorien}

$\mathrm{Zu}$ derartigen qualitativen Innovationen, welche sich außerhalb des Menschen im Tierreich nicht finden und welche sich nur im Kontext der genannten strengen Verflechtung mehrerer Strukturebenen ausbilden können, sind schließlich die vielfältigen sowie bedeutungsreichen grammatischen Kategorien und Funktionen menschlicher Sprachen zu zählen. Eine erschöpfende Einführung oder auch nur eine abschließende Skizze kann freilich im gegebenen Kontext nicht angeboten werden, sodass stattdessen ein weiteres Beispiel exemplarisch diskutiert werden soll. In dieser Diskussion werden einige Kategorien wie die vormals genannten Wortarten außen vor gelassen und stattdessen ausgewählte sprachlich-strukturelle Kodierungsmuster inhaltlich expliziert, ohne dass jedoch fachliche Begrifflichkeiten herangezogen oder die jeweiligen strukturellen Eigenschaften weiter erläutert werden sollen.

Er wäre nur am Trotzen gewesen.

Beispiel (9) zeigt einen denkbar unscheinbaren Satz, welcher zugunsten der vorliegenden Veranschaulichung als Antwort auf die Frage „Warum hast du gestern deinen Sohn nicht in die Spielgruppe gebracht?“ angesehen werden 
soll. Auf einschlägige Fachterminologie soll wie eben angemerkt und im Bisherigen geschehen weitestgehend verzichtet werden, um den $\mathrm{zu}$ veranschaulichenden Punkt mit größtmöglicher Eingängigkeit vorbringen zu können. In diesem Sinne ist sich nun die Frage zu stellen, welche Informationen in jenem Satz aufzufinden sind und innerhalb einer Konversation vermittelt werden, ohne dass innerhalb dieser Gesprächssituation vom Produzenten oder vom Rezipienten explizit hierüber nachgedacht werden müsste.

Erstens definiert jene Äußerung sofort, auf wen sie sich bezieht - nicht auf den Gesprächspartner, die äußernde Person, sonstige Drittpersonen oder Gruppen, sondern auf den vorgenannten Sohn. In Bezug auf diesen reicht die gegebene Form dazu aus, eine nahtlose Kontinuität des Dialogs aufrechtzuerhalten. Wie bedeutend diese eindeutige Referenz tatsächlich ist, wird womöglich erst klar, sobald darauf hingewiesen wird, dass nicht-menschliche Kommunikation einen derartigen Fokus nicht zulässt. Dort ist ein Ruf entweder als von einem derartigen Informationsgehalt frei aufzufassen oder so zu interpretieren, dass er bestenfalls eine innerhalb imperativen Kommunikationskontexten inhärent gegebene, unscharfe du-ich-Beziehung impliziert. Ein jedes Individuum, welches einen Ruf wahrnimmt, muss damit eigenständig damit beginnen, die vom Rufenden intendierte bzw. implizierte Referenz in der Umwelt zu suchen. Anders ist ein Bezug zwischen Äußerung und Gemeintem nicht möglich. Erst Sprachexperimente schaffen auch nur im Entferntesten eine Vergleichbarkeit, indem dort für ausgewählte Individuen quasi Eigennamen definiert werden können. Doch auch hier sind starke Limitationen gegeben, denn Eigennamen können die grammatische Perspektivierung natürlicher Sprachen nicht umfänglich ersetzen. Damit ist in jedem Fall die Offenkundigkeit der Information, wer an einer Situation beteiligt ist - und ob als Individuum bzw. als Gruppe - , in dieser Form lediglich in natürlichen Sprachen gegeben.

Zweitens legt die gegebene Formulierung sogleich dar, dass es sich nicht um eine faktische Aussage handelt. Stattdessen verschiebt sich dasjenige, über das gesprochen wird, in eine hypothetische Realität - im Sinne der Frage, was gewesen wäre, wenn sich die Situation, über die gesprochen wird, anders entwickelt hätte. Diese Versetzung von der unmittelbaren, faktischen Realität in eine irreale Welt findet sich wenig überraschend nicht innerhalb nichtmenschlicher Kommunikation und stellt eine der leistungsfähigsten funktionalen Komponenten menschlicher Sprache dar. Handlungsoptionen abzuschätzen und damit in begrenztem Maße Urteile $\mathrm{zu}$ möglichen Welten $\mathrm{zu}$ fällen stellt durchaus eine Befähigung des nicht-menschlichen Bereichs dar, jedoch fehlt dagegen die Explikation fiktiver Situationen und alternativer Geschehnisse bzw. Handlungen in kommunikativer Form vollständig. In der Tat fundiert neben 
Weiterem gerade diese Befähigung die menschliche kognitive, soziale, kulturelle und technologische Alleinstellung maßgeblich, denn sie erlaubt eine leistungsfähige Langzeitplanung sowohl von Individuen als auch zwischen Individuen.

Drittens und in ähnlicher Weise beschreibt jenes Beispiel nicht die temporal unmittelbar erlebte Realität. Tatsächlich wird der Diskurs nicht nur wie soeben beschrieben in eine hypothetische Realität versetzt, sondern darüber hinaus in den Kontext eines bereits vergangenen Zeitpunkts. Nun legt die einschlägige Forschung nahe, dass manche nicht-menschliche Tiere durchaus rudimentäre Formen eines episodischen Gedächtnisses aufweisen (vgl. bspw. Templer \& Hampton 2013 sowie Beran et al. 2016), jedoch besitzt dieses anscheinend keine mit dem Menschen vergleichbare propositionale gedankliche Struktur und insbesondere existiert in nicht-menschlichen Kommunikationssystemen keine Möglichkeit der Versetzung eines kommunikativen Aktes in eine ebensolche vergangene Situation respektive innerhalb alternativer kommunikativer Kontexte auch in zukünftige Situationen. Dass hierin ein weiterer Grundstein der menschlichen Alleinstellung besteht, liegt nahe - und diese Aussage wird zusätzlich untermauert, wenn die vorliegende sprachliche Dimension durch die zuvor erläuterten, die nachfolgend vorgestellten und weitere, im gegebenen Kontext nicht diskutierte Dimensionen fruchtbar erweitert wird.

Viertens kodiert der Beispielsatz weiterhin, dass jener eindeutig referenzierte Sohn innerhalb der aufgemachten hypothetischen sowie vergangenen Realität in einer fortwährenden, zeitlich ausgedehnten Handlung involviert gewesen wäre - anstatt in einer singulären, kurzfristigen Handlung - und fünftens wird deutlich, dass dieser Sohn als der Agierende der explizierten Handlung zu sehen ist und nicht als passiv beteiligtes Ziel bzw. Objekt einer Handlung oder Situation. Derlei Klärungen mögen auf den ersten Blick weniger substanziell erscheinen als die zuvor diskutierten, jedoch erhöhen auch diese sprachlichen Dimensionen durch die involvierten Perspektivierungen die Flexibilität und Leistungsfähigkeit sprachlicher Kommunikation sowie eines expliziten, monologischen Überlegens. Freilich liegen hierbei zwei weitere Dimensionen vor, welche weder in der freien Wildbahn noch in experimenteller Umgebung auf nicht-menschliche Kommunikation zutreffen.

All diese Kategorien konstruieren im gegebenen Beispiel gemeinsam mit den eigentlichen lexikalischen Einheiten eine komplexe und anspruchsvolle Aussage, welche wiederum in einer sprachlichen und situativen Umgebung $\mathrm{zu}$ verorten ist - d.h. in diesem Fall innerhalb eines Dialogs mit fortlaufenden Themen sowie Personen und in einem konkreten Kontext, welcher sprachliche Äußerungen bzw. die Form und Bedeutung sprachlicher Äußerungen zusätzlich 
informiert. Dass der Satz dabei intuitiv fließend und mühelos verstanden werden kann, zeigt nicht eine Simplizität von Sprache an, sondern belegt vielmehr die erstaunliche Leistungsfähigkeit der menschlichen Sprachbefähigung. $\mathrm{Zu}$ betonen ist hierbei erstens, dass nicht-menschliche Kommunikation prinzipiell nicht in der Lage ist, auch nur einen Teil dieser sprachlichen Dimensionen angemessen abzubilden, und zweitens, dass hier keinesfalls eine abschließende Erläuterung derartiger sprachlicher Phänomene gegeben wurde, sondern lediglich ein vager und beispielhafter Einblick anhand eines konkreten Beispiels.

\subsection{Eine notwendige Anmerkung zur Komplexität natürlicher Sprachen}

Auf einen kleinen Einwand ist an gegebener Stelle noch einzugehen, um die gegebene Diskussion abzusichern: Eine große Zahl bekannter Sprachen beinhalten - wie anhand des obigen Beispiels dargestellt - strukturell offenkundige grammatische Kategorien, während wiederum andere Sprachen den Informationsgehalt mancher Kategorien auf den ersten Blick zu missen scheinen. Dem ist jedoch nicht zu folgern, dass zwischen natürlichen Sprachen Hierarchien der Komplexität festzustellen seien oder dass solche Sprachen mit scheinbar fehlenden grammatischen Kategorien näher mit nicht-menschlicher Kommunikation vergleichbar wären als beispielsweise das Deutsche, denn in aller Regel existieren die entsprechenden sprachlichen Dimensionen in jenen Sprachen durchaus - sie werden lediglich, wenn aus Sicht klassischer Grammatiken betrachtet, in ungewohnter Weise realisiert. Ganz in diesem Sinne sollte man „einer Sprache eine grammatische Kategorie nicht vorschnell absprechen, nur weil sie nicht in den gewohnten Mustern transparent wird“ (Leiss 1992: 27). In diesem Sinne zeigen sich natürliche Sprachen als wandlungsfähig sowie ressourcenreich und vermögen es im Verlauf von historischen Zeiträumen, Funktionen von einer grammatischen Kategorie in eine andere $\mathrm{zu}$ übertragen oder auch lexikalische respektive pragmatische Mittel zu konventionalisieren, um für die Dekonstruktion einer grammatischen Kategorie eine angemessene Kompensation anzubieten.

Um einen solchen Wandel knapp zu veranschaulichen, sei darauf verwiesen, dass das heutige Standarddeutsche - und hier muss die vorliegende Diskussion wenigstens kurzfristig gezwungenermaßen auf Fachterminologie zu- 
rückgreifen - die grammatische Kategorie des Aspekts ${ }^{35}$ im Gegensatz zu historisch älteren Sprachstufen vermisst. Einerseits findet die heutige Sprachverwendung zur Kompensation des Wegfalls des grammatischen Aspekts lexikalische Mittel wie in „Ich koche gerade.“ oder mit dem am-Progressiv wie in „Ich bin am Kochen." und andererseits zeigt Leiss (2000), dass sich zwischen dem Althochdeutschen und dem Neuhochdeutschen die dem Letzteren typische Artikelverwendung herausbildet, um die Kategorie der Definitheit, welche im Althochdeutschen noch im Kontext des Aspekts kodiert wurde, von der verbalen Umgebung in die nominale zu übertragen.

Die nachfolgenden Beispiele (10a) bis (10c) suchen die Artikelverwendung des Deutschen ganz allgemein zu veranschaulichen. Dies bietet sich im Nachgang zum eben Vorgestellten an und unterstreicht zusätzlich die Diffizilität natürlicher Sprachen, welche im gegebenen Kontext ohnehin verdeutlicht werden soll. (10a) verzichtet auf einen Artikel und beschreibt damit ohne inhärenten Start- oder Endpunkt den Prozess des Biertrinkens zum temporal vergangenen Zeitpunkt, den die Aussage anspricht. (10b) gibt der Handlung wenigstens einen Endpunkt, denn es wurde nicht vage Bier getrunken, sondern ein konkretes Bier zu Ende getrunken, wobei die Verwendung des Artikels indefinit verbleibt - d.h. die Äußerung kodiert die unbestimmte Natur des Bieres in dem Sinne, dass eine konkretere Angabe zum Bier für den sprachlichen Diskurs nicht von Wichtigkeit ist. (10c) dagegen verwendet den definiten Artikel, weil nun die Bestimmtheit des Bieres anscheinend von Wichtigkeit im gegebenen sprachlichen Kontext ist. So könnte beispielsweise der Fall sein, dass eine Person gegenüber ihrem Mitbewohner explizit anmerkte, dass sich noch ein offenes Bier im Kühlschrank befindet - mit der Implikation, dass es zeitnah zu trinken sei. In einem solchen Kontext macht die spätere Verwendung von (10c) gegenüber (10a) oder (10b) dahingehend Sinn, dass nur hier auf das vormals bereits angesprochene, konkrete Bier Bezug genommen wird. Dieser Abruf vorhandener Information begründet erst die Sinnhaftigkeit dieser Aussage, welche ansonsten kontextlos und irritierend verbliebe.

35 Im Gegensatz zur grammatischen Kategorie des Tempus, welche den Zeitpunkt einer Handlung bzw. eines Vorgangs in Relation zu einem fixierten Zeitpunkt - beispielsweise zu demjenigen des Sprechers - kodiert und damit die zeitliche Beziehung zwischen zwei Ereignissen zum Ausdruck bringt, definiert Aspekt die Handlung bzw. den Vorgang hinsichtlich Ausdehnung oder Vollendung im Verhältnis zum betrachteten Zeitpunkt/-raum. Um dies zusätzlich zu verdeutlichen, sei sich ein Zeitstrahl von aufeinanderfolgenden Handlungen vorzustellen. Tempus verortet eine Handlung bzw. einen Vorgang auf diesem Zeitstrahl, während Aspekt die Ausdehnung und Abgeschlossenheit an respektive rund um dieser Verortung zu definieren vermag. 

a. Ich habe Bier getrunken.
b. Ich habe ein Bier getrunken.
c. Ich habe das Bier getrunken.

\subsection{Linguistisch fundierte Einsichtmöglichkeiten in die Evolution menschlicher Sprache}

Zum Abschluss des vorliegenden Kapitels seien wie angekündigt noch linguistische Perspektiven genannt, anhand derer die phylogenetische Entstehung von Sprache potenziell nachgezeichnet werden kann. Generell bietet sich diese Möglichkeit, indem entweder die zeitgenössische Konstruktion neuartiger sprachlicher Phänomene untersucht wird, sobald sich diese Gelegenheit innerhalb entsprechender Kontexte bietet, oder indem auch die Konstitution natürlicher Sprachen bzw. gegebenenfalls deren Dekonstruktion betrachtet wird. Ein erster Ansatz betrifft die Ontogenese von Sprache: Der Spracherwerb im Kindesalter erfolgt bis zu einem Alter von etwa zwei Jahren in mehrerlei Hinsicht in vergleichbarer Weise wie der Erwerb von Kommunikationssystemen in Sprachexperimenten mit Menschenaffen und wie bereits angemerkt zeigen sich nichtmenschliche Menschenaffen insbesondere auf der Seite des Rezipienten als durchaus leistungsfähig (vgl. bspw. nochmals Savage-Rumbaugh et al. 1993). In Folge tritt der Erstspracherwerb als eine vielversprechende Analogie zur frühmenschlichen phylogenetischen sprachlichen Entwicklung in Erscheinung.

Zusätzlich existieren auch innerhalb ontogenetisch voll ausgebildeter moderner Sprachen produktive Schablonen, welche in protosprachlichen Systemen möglicherweise bereits Anwendung fanden. Hierzu zu zählen ist beispielsweise die Kompositabildung, welche von den Befähigungen nichtmenschlicher Menschenaffen nicht weit entfernt zu sein scheint, bzw. allgemeiner Kompositionalität, welche wie zuvor diskutiert in das Befähigungsspektrum einiger Arten fällt. Jackendoff (1999) sowie daran anschließend beispielsweise Progovac (2015 und 2016) nennen diese und weitere Phänomene entsprechend auch sprachliche lebende Fossile (,living fossils“). Auch sind Kombinationen zwischen der oralen und der gestisch-deiktischen Modalität zu erwähnen, denn auch diese stehen in Reichweite nicht-menschlicher Befähigungen und schaffen Potenzial für leistungsfähige protosprachliche Systeme (Greenfield, Lyn \& Savage-Rumbaugh 2008).

Eine weitere Möglichkeit der Einsicht in den sukzessiven Aufbau sprachlicher Systeme, welche sich womöglich auf die Phylogenese anwenden lassen, 
bieten Phänomene wie Homesign, Pidgin und Kreol - d.h. Situationen, in denen entweder ein normativer Erstspracherwerb nicht stattfinden kann oder neuartige sprachliche Systeme entstehen (vgl. bspw. Brentari \& Coppola 2013 sowie Brentari \& Goldin-Meadow 2017), denn derartige Systeme entstehen nie spontan in vollständiger Weise, sondern bilden sich in sukzessiver Weise und mitunter über mehrere Generationen heraus (vgl. bspw. die nicaraguanische Gebärdensprache und einschlägige Untersuchungen; siehe Senghas et al. 2005 sowie Kocab et al. 2016 neben den Vorgenannten für eine allgemeinere Perspektive). Zuletzt sind sowohl gewisse pathologische Phänomene als auch unglückliche Fälle sprachlicher Deprivation Ansätze für fruchtbare Einsichten in die Konstruktion und Konstitution der internen, umfänglich hierarchischen Struktur von Sprache.

All diesen Untersuchungen liegt freilich die linguistische Forschung als zentraler Betrachtungsansatz zugrunde. Sowohl in dieser direkten Hinsicht als auch an interdisziplinären Schnittstellen wie bei Untersuchungen zu Befähigungen nicht-menschlicher Tiere sollte damit beim Forschenden ein ausreichender Wissensstand zur linguistischen Perspektive gegeben sein, um eine angemessene Analyse überhaupt erst zu ermöglichen. Mithin macht es Sinn, ein Wissen aufzubauen, welches das hier Präsentierte übersteigt. Um den Grenzen der vorliegenden Arbeit jedoch gerecht zu werden und den weiteren Anliegen dieses Vorhabens ausreichend Raum geben zu können, sei hiermit die einführende Skizze zur Rolle der Sprachwissenschaften innerhalb der Sprachursprungsforschung als abgeschlossen zu sehen. Wie zuvor dargelegt besteht mit Abschluss dieses Abschnitts und mit dem Gesamtkontext der vorangegangenen Einführungen eine gerundete, jedoch durchaus oberflächliche Fundierung für nachfolgende, weiterführende Diskussionen. 
